Third Department of Internal Medicine,

Hyogo College of

Medicine,

Mukogawa-cho 1-1, Nishinomiya, Hyogo

663-8501, Japan

T Yamamoto

Y Moriwaki

K Matsui

S Takahashi

K Higashoni

Department of

Immunology and

Medical Zoology,

Hyogo College of

Medicine

H Tsutsui

T Yoshimoto

K Nakanishi

Laboratory of Host Defenses, Institute for Advanced Medical

Sciences, Hyogo

College of Medicine

H Okamura

Department of

Pediatrics, Keio

University School of

Medicine, Tokyo, Japan

Y Kurosawa

Department of

Pediatrics, The

Kitasato Institute

Hospital, Tokyo, Japan

$S$ Yamaguchi

Department of Safety Research on Biologics, National Institute of Infectious Disease,

Tokyo, Japan

Y Sasaki

Correspondence to: Dr Yamamoto.

Accepted 13 March 1999

Table 1 Laboratory data in PNP deficient patient

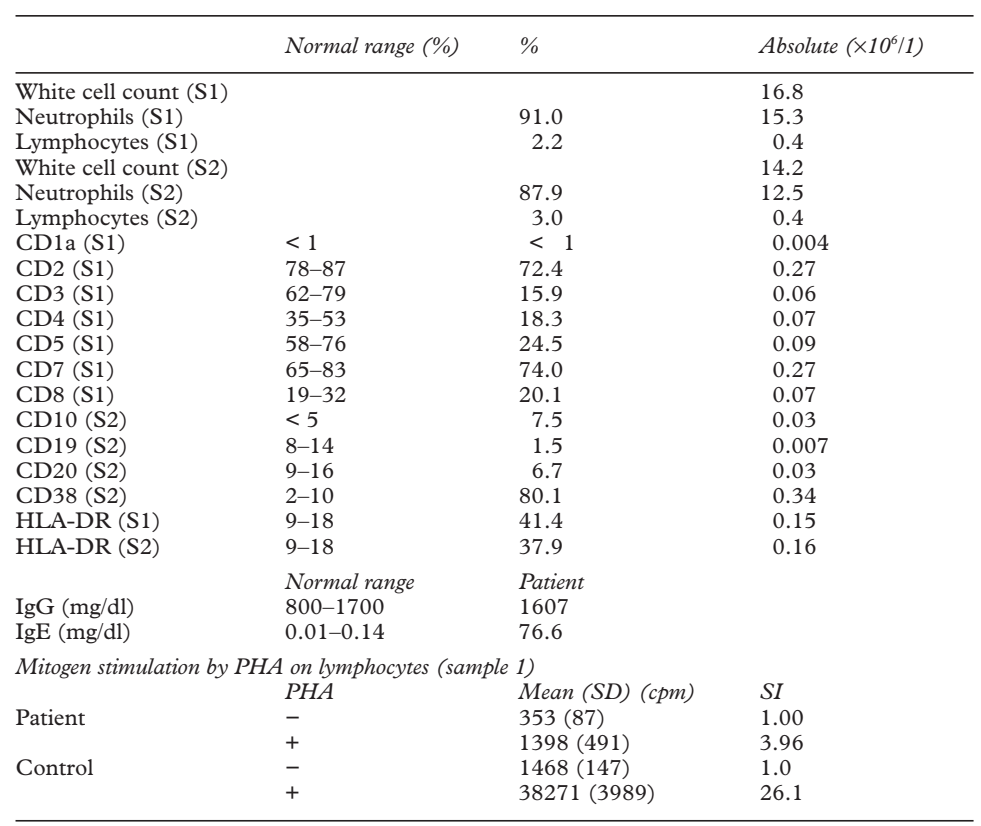

S, Sample, PHA, phytohaemagglutinin, cpm, counts per minute, SI, stimulation index. Shinya Yamaguchi, Yuko Sasaki, Kazuya Higashino

Keywords: purine nucleoside phosphorylase deficiency; interferon- $\gamma$ inducing factor; cytokines

A deficiency of purine nucleoside phosphorylase (PNP) has sometimes been found in patients with severe susceptibility to infection. In patients with PNP deficiency, deoxyguanosine, one of the substrates of PNP, increases in plasma and urine, and dGTP is detected in erythrocytes, suggesting that an increase in the concentration of deoxyguanosine leads to accumulation of dGTP in cells, which may be toxic for $\mathrm{T}$ cells. ${ }^{1} \mathrm{~B}$ and $\mathrm{T}$ cell functions have been investigated in patients with PNP deficiency, ${ }^{1}$ while cytokines secreted into blood

\title{
High IL-18 (interferon- $\gamma$ inducing factor) concentration in a purine nucleoside phosphorylase deficient patient
}

Tetsuya Yamamoto, Yuji Moriwaki, Kiyoshi Matsui, Sumio Takahashi, Hiroko Tsutsui, Tomohiro Yoshimoto, Haruki Okamura, Kenji Nakanishi, Yoshihiro Kurosawa,

\begin{abstract}
The plasma concentration of IL-18 (interferon- $\gamma$ inducing factor) was severely increased in a 3 year old boy with purine nucleoside phosphorylase (PNP) deficiency. The presence and activity of IL-18 were confirmed by immunoblotting and bioassay, respectively. These results suggest that IL-18 may be abundantly produced and secreted into plasma by PNP deficient macrophages in PNP deficiency.

(Arch Dis Child 1999;81:179-180)
\end{abstract} determine the serum concentration of cytokines. Leukocyte counts, lymphocyte subset, immunoglobulin levels, and mitogen response analyses were conducted using standard laboratory methods. IL-18 and other cytokine immunoassays were performed as previously described $^{3}$ or according to manufacturer's instructions (Otsuka Assay Research Laboratory, Tokushima, Japan).

Plasma IL-18 concentration was also determined by induction of interferon- $\gamma$ (IFN $\gamma$ ) production by $\mathrm{T}$ cells from the tonsil of a tonsillectomy patient cultured at $2 \times 10^{6} \mathrm{cells} / \mathrm{ml}$ for four days with IL-12 (100 or $200 \mathrm{ng} / \mathrm{ml}$ ) and various concentrations of IL-18 or diluted plasma (1:4) from the patient or normal control either in the presence or absence of anti-IL-18 antibody. IFN $\gamma$ in the culture supernatants was measured by specific enzyme linked immunosorbent assay (ELISA) (Bio Source International, Camarillo, California, USA).

\section{Results}

Table 1 shows immunological data. The plasma concentration of IL-18 was $216 \mathrm{ng} / \mathrm{ml}$ 


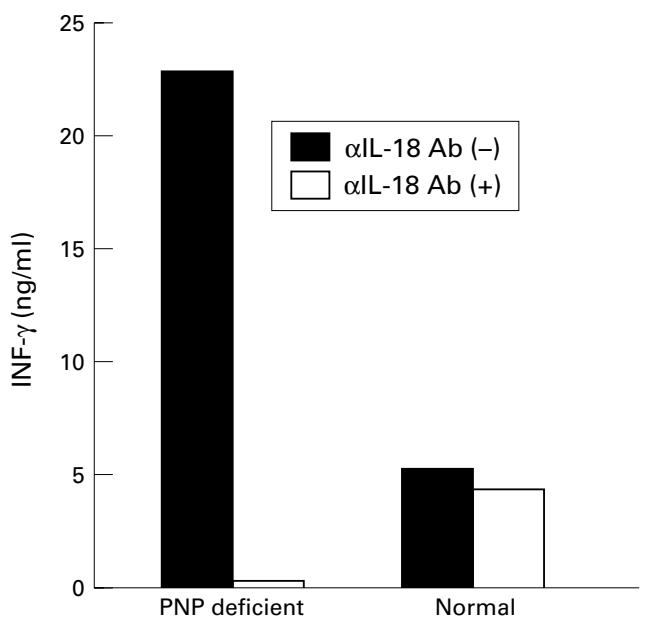

Figure 1 IFN $\gamma$ production by human $T$ cells treated with IL-12 treated either with plasma of the PNP deficient patient or from a normal control. (Ab, antibody.)

(sample 3) and $66 \mathrm{ng} / \mathrm{ml}$ (sample 4). These concentrations are extraordinarily high compared with those in patients with various infectious diseases, rheumatoid arthritis, gout, and normal subjects $(<10,<2,<0.5$, and $<0.2 \mathrm{ng} / \mathrm{ml}$, respectively). The plasma concentrations of other cytokines were measured using sample 3. The plasma concentration of IFN $\gamma$ was raised $(84 \mathrm{pg} / \mathrm{ml}$, normal range $<20$ ). In contrast, the plasma concentrations of IL-4, IL-5, TNF $\alpha$, IL-1 $\alpha$, IL-1 $\beta$, IL-6, IL-2, IL-8, and granulocyte macrophage colony stimulating factor (GM-CSF) were all normal in the patient, although M-CSF $(1843 \mathrm{pg} / \mathrm{ml}$, normal < 1800) was slightly raised.

An immunoblot analysis of plasma with anti-IL-18 monoclonal antibody showed an $18 \mathrm{kDa}$ band and other non-specific bands in the patient with PNP deficiency, while it showed only non-specific bands in a healthy subject (data not shown).

The IFN $\gamma$ response $(23.6 \mathrm{ng} / \mathrm{ml})$ in the biological assay using the patient's plasma (sample 4) corresponds to a value of $72 \mathrm{ng} / \mathrm{ml}$ for plasma IL-18 derived from a standard curve (not shown), a value similar to that found by immunoassay (fig 1). The addition of antiIL-18 antibody to the biological assay system completely blocked the secretion of IFN $\gamma$ from IL-12 treated T cells in the patient's plasma (fig 1).

\section{Discussion}

We have demonstrated decreased CD3 positive and CD19 positive lymphocyte numbers, and mitogen responses in our patient. However, plasma concentrations of $\operatorname{IgG}$ and $\mathrm{IgE}$ were normal, suggesting PNP deficiency preferentially affected $\mathrm{T}$ cell function in this case (table 1). The most intriguing finding in this study was the extraordinarily high plasma concentrations of IL-18. The immunoblot analysis of IL-18 in PNP deficient plasma showed that the detected cytokine was an $18 \mathrm{kDa}$ polypeptide consistent with its mature form. The biological assay of IL-18 in PNP deficient plasma showed that the cytokine was biologically active. This increase may have been induced by the excessive action of natural killer (NK) cells and $\mathrm{B}$ cells in our patient who lacked $\mathrm{T}$ cell immune function. ${ }^{4}$

In patients with PNP deficiency, IL-18 may be excessively synthesised as precursor protein and actively processed to mature protein by caspase-1. ${ }^{4}$ Although IL-18 seems to play an important role in defence against infection with microbes that activate macrophages and NK cells to produce IL-12 and IL-18, we suggest that the very high plasma IL-18 in our patient was not a response to infection as it was much greater than levels in patients with inflammatory diseases, such as rheumatoid arthritis, and other infectious diseases. Furthermore, IL-1 $\beta$ and IL-6, which are also major products of macrophages and similarly induced by infectious diseases, were not raised in the plasma of this patient. We propose that in PNP deficiency the production and secretion of IL-18 is increased perhaps because PNP deficient $T$ cells fail to regulate the macrophages or NK cells that produce the cytokine.

We are grateful to Yasuko Hyodo and Kouji Tominaga for excellent technical assistance. This work was supported in part by a grant from the Gout Research Foundation of Japan.

1 Fairbanks LD, Taddeo A, Duley JA, Simmonds AN. Mechanisms of deoxyguanosine lymphotoxicity. Human thymocytes, but not peripheral blood lymphocytes, accumulate deoxy-GTP in conditions simulating purine nucleoside phosphorylase deficiency. F Immunol 1990;144:485-91.

2 Sasaki Y, Iseki M, Yamaguchi S, et al. Direct evidence of autosomal recessive inheritance of Arg-24 to terminal codon in purine nucleoside phosphorylase gene in a family with a severe combined immunodeficency patient. Hum Genet 1998;103:81-5.

3 Taniguchi M, Nagaoka K, Kunikata T, et al. Characterization of anti-human interleukin-18 (IL-18) interferon- $\gamma$ inducing factor (IGIF) monoclonal antibodies and their application in the measurement of human IL-18 by ELISA. f Immunol Methods 1997;206:107-13.

4 Okamura H, Tsutsui H, Kashiwamura S, Yoshimoto T, Nakanishi K. Interleukin-18: a novel cytokine that augments both innate and acquired immunity. Adv Immunol 1998;79:281-312. 